\title{
Nasal Continuous Positive Airway Pressure in Bronchiolitis: A Randomized Controlled Trial
}

\author{
Sandeep Narayan Lal, Jaspreet Kaur, Pooja Anthwal, Kanika Goyal, Pinky Bahl and Jacob M PuliYel \\ From the Department of Pediatrics, St. Stephens Hospital, Tis Hazari, Delhi, India. \\ Correspondence to: Dr Sandeep Narayan Lal, Department of Pediatrics, St Stephens Hospital, Tis Hazari, Delhi 110054, India. \\ sandeep.nlal@gmail.com \\ Received: October 28, 2016; Initial review: February 14, 2017; Accepted: September 09, 2017.
}

Objective: To evaluate the efficacy of nasal continuous positive airway pressure (nCPAP) in decreasing respiratory distress in bronchiolitis.

Design: Randomized controlled trial.

Setting: Tertiary-care hospital in New Delhi, India.

Participants: 72 infants (age $<1 \mathrm{y}$ ) hospitalized with a clinical diagnosis of bronchiolitis were randomized to receive standard care, or nCPAP in addition to standard care, in the first hour after admission. 23 parents refused to give consent for participation. 2 infants did not tolerate nCPAP. nCPAP was not tolerated or the distress increased, the infant was switched to standard care. Analysis was done on intention-to-treat basis.
Intervention: The outcome was assessed after 60 minutes. If

Main outcome measures: Change in respiratory rate, Silverman-Anderson score and a Modified Pediatric Society of New Zealand Severity Score.

Results: 14 out of 32 in nCPAP group and 5 out of 35 in standard care group had change in respiratory rate $\geq 10(P=0.008)$. The mean (SD) change in respiratory rate $[8.0$ (5.8) vs 5.1 (4.0), $P=0.02]$ in Silverman-Anderson score $[0.78(0.87)$ vs $0.39(0.73)$, $P=0.029]$ and in Modified Pediatric Society of New Zealand Severity Score $[2.5$ (3.01) vs. 1.08 (1.3), $P=0.012]$ were significantly different in the nCPAP and standard care groups, respectively.

Conclusion: nCPAP helped reduce respiratory distress significantly compared to standard care.

Keywords: $n C P A P$ Ventilation, Respiratory distress, Respiratory Syncytial Virus, Wheezing infant.

Published online: September 26, 2017. PII:S097475591600096

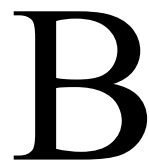
ronchiolitis is characterized by edema, mucus secretions and damage of airway epithelium with necrosis, causing airflow obstruction and distal air trapping, atelectasis and a ventilation perfusion mismatch that leads to hypoxemia and increased work of breathing [1,2]. Clinical improvement with significant falls in respiratory rate, pulse rate and pressure of carbon dioxide $\left(\mathrm{pCO}_{2}\right)$ has been reported with the use of Continuous positive airway pressure (CPAP) in Bronchiolitis $[3,4]$. In this study, we evaluated whether CPAP is clinically beneficial in infants with moderate-tosevere bronchiolitis during the first hour of treatment.

\section{MeTHODS}

This randomized control trial was conducted at St. Stephen's Hospital (SSH), Delhi, between November 2014 and March 2016. All patients who were hospitalized with a clinical diagnosis of acute bronchiolitis were eligible for inclusion in the study. Bronchiolitis was defined as respiratory distress (respiratory rate $\geq 50 / \mathrm{min}$ ) in an infant aged 1 month to 1 year, along with wheezing on auscultation and hyper- inflated lung. Infants who were in imminent need of ventilator support were excluded.

Approval for the trial was obtained from the hospital's research ethics committee. Informed consent of the parents of eligible children was obtained. Patients were then randomized to receive CPAP or standard care. Randomization into the two groups was done in blocks of 8 using computer software (www.randomization.com) seed number 14184) and allocation to the groups was done using sequentially-numbered opaque sealed envelopes. All patients received standard care, which included maintenance of adequate hydration and oxygenation, while the intervention group received bubble CPAP in addition to the standard care. Bubble CPAP was delivered in the pediatric ward with a Gregory circuit $[5,6]$. Oxygen saturation was noted before starting treatment and oxygen was supplemented if the saturation was $<92 \%$. Those in the intervention group received oxygen through the bubble CPAP system while the standard care group received oxygen through mask or hood. All children were monitored continuously during 
the study period. The protocol mandated that CPAP would be stopped if the distress of the patients increased (defined as increase in RR of more than 10), or if the infant was very restless and not tolerating CPAP. If the saturation fell below $90 \%$ in either group and persisted to be so for more than $>15$ minutes despite oxygen supplementation, the child was considered for mechanical ventilation. Treatment decisions were based on the judgment of the treating physician but the changes made were documented.

The respiratory rate (RR), Silverman-Anderson score, and a Modified Pediatric Society of New Zealand severity score (MPSNZ-SS) were assessed before starting treatment and at 1 hour following the start of treatment. Respiratory rate was assessed by the staff nurse on duty. The respiratory rate was counted for 60 seconds continuously or in 2 blocks of 30 seconds. The Silverman-Anderson score was assessed by the doctor on duty. The MPSNZ-SS was also assessed by the doctor on duty based on the history and the clinical parameters. It was evaluated by modifying the Pediatric Society of New Zealand (PSNZ) severity scoring system that is based on six parameters (respiratory rate, chest wall indrawing, nasal flaring or grunting, feeding, history of behaviour, cyanosis) [7]. The original PSNZ guidelines used cyanosis as a criteria but we substituted it with oxygen saturation. Each of these parameters was assigned a score of 1 to 3 with increasing severity and a final score was calculated.

The primary outcome was to compare the change in respiratory rate after the first hour of treatment among the two groups. A decrease in respiratory rate of 10 or more was considered clinically significant difference. The secondary outcomes were the change in SilvermanAnderson score and the MPSNZ-SS.

A previous study reported a $24 \%$ change in respiratory rate (RR) in the study group compared to negligible change in the standard care group [4]. For a

TABLE I BASELINE CHARACTERISTICS IN THE STUdY GROUPS

\begin{tabular}{llll}
\hline Characteristics & $\begin{array}{l}\text { Bubble CPAP } \\
\text { group }(n=36)\end{array}$ & $\begin{array}{l}\text { Standard care } \\
\text { group }(n=36)\end{array}$ & Pvalue \\
\hline Age (mo) & $4.0(2.6)$ & $4.7(3.1)$ & 0.36 \\
Male gender, No. (\%) & $26(72.2)$ & $28(77.8)$ & 0.59 \\
Respiratory rate & $70(11)$ & $67(7)$ & 0.17 \\
SA score & $4.2(2.1)$ & $3.8(1.7)$ & 0.74 \\
MPSNZ-SS & $12.4(2.7)$ & $11.7(2.4)$ & 0.21 \\
\hline
\end{tabular}

SA: Silverman Anderson; MPSNZ-SS: Modified Pediatric Society of New Zealand Severity Score; All values in mean (SD). type I error of 0.05 and a type II error of 0.2 , we calculated that a sample size of 72 was needed for a 1:1 ratio of standard care to CPAP.

Statistical analysis: Normality of data was tested by Kolmogorov-Smirnov test. If the normality was rejected, non parametric tests were used. Respiratory rate (RR) at admission, change in respiratory rate after 1 hour of treatment $(\Delta R R)$, and modified Pediatric Society of New Zealand Severity Score (MPSNZ-SS) at admission were normally distributed and were compared using independent $\mathrm{T}$ test and non-parametric data as age in months, RR at the end of 1 hour, Silverman-Anderson (SA) score, MPSNZ-SS at the end of 1 hour, and the change in MPSNZ-SS ( $\triangle$ MPSNZ-SS) were compared using Mann-Whitney test between two groups. Change in $\mathrm{RR}(\Delta \mathrm{RR}<10$ and $\geq 10)$ was compared using chi square test between the two groups. Wilcoxon ranked sum test was used to compare RR, SA Score and MPSNZ-SS within groups across follow-up.

\section{RESUlTS}

The study was continued till 72 patients were recruited. A total of 117 children were hospitalized with a provisional diagnosis of bronchiolitis during this period. Fig. 1 depicts the flow of participants in the study.

The baseline characteristics of the two groups are described in Table I. There was no significant difference between the two groups. Table II shows the mean change in respiratory rate, Silverman-Anderson score and MPSNZ-SS in the two groups after 1 hour of treatment. There was statistically significant improvement in RR, SA score and MPSNZ-SS in the bubble CPAP group when compared to standard care group.

In the bubble CPAP group, 14 out of 32 patients showed clinically significant improvement $(\Delta R R \geq 10)$, whereas 5 out of 35 patients had clinically significant improvement in the standard care group $(P=0.008)$. Three patients needed mechanical ventilation within 1 hour of starting treatment as the distress progressively worsened. Of these, two were from the CPAP group and

TABLE IICOMPARISON OF CHANGE IN RESPIRATORY DiSTRESS IN THE Two Groups After 1 Hour

\begin{tabular}{lllr}
\hline Parameter & Bubble CPAP & Standard care & Pvalue \\
\hline Respiratory rate & $8(6)$ & $5(4)$ & 0.02 \\
SA score & $0.78(0.87)$ & $0.39(0.73)$ & 0.03 \\
MPSNZ-SS & $2.5(3.0)$ & $1.1(1.3)$ & 0.01 \\
\hline
\end{tabular}

SA:Silverman-Anderson; MPSNZ-SS: Modified Pediatric Society of New Zealand Severity Score; All values in mean (SD). 


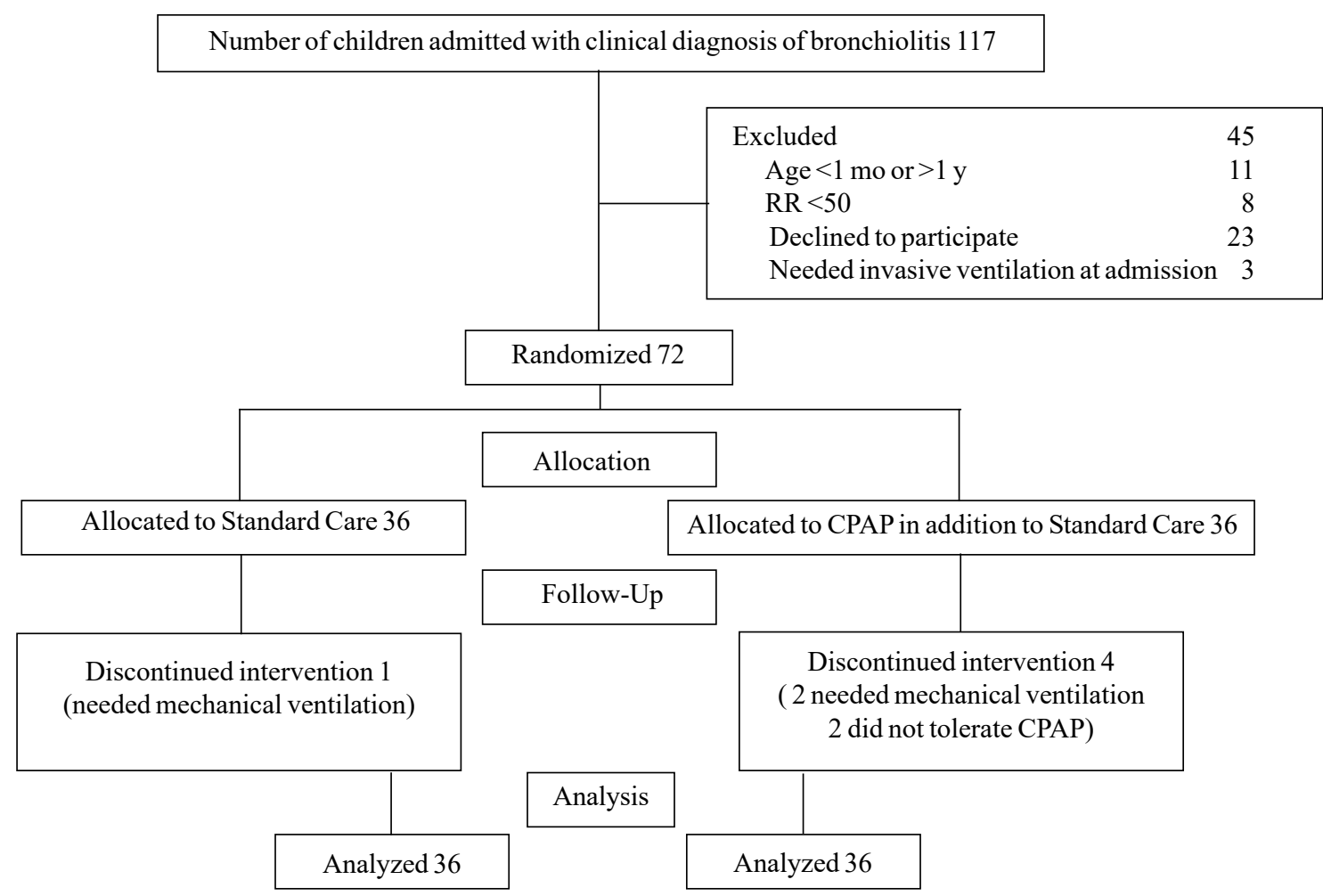

Fig. 1: Study flow chart.

one was from standard treatment group. CPAP had to be stopped in two children before 1 hour, as they were very restless and became more agitated following start of CPAP. Table II compares the outcomes between two groups.

\section{Discussion}

In this randomized controlled trial, we documented that CPAP significantly reduced the respiratory rate in comparison to standard care in infants with bronchiolitis. The Silverman-Anderson score and MPSNZ-SS also showed significant improvement with CPAP during the first hour of treatment.

Bronchiolitis is a clinical diagnosis with limited role of laboratory and radiological evaluation $[1,8]$. We included all infants during the bronchiolitis season with respiratory distress and a respiratory rate $\geq 50$, at the time of admission. As pneumonia is also common in this age group and it also presents with similar symptoms initially, it is possible that few patients included in the study were cases of pneumonia. Ours was an open label study and could have been influenced by observer bias. Patient parameters were assessed by the doctors and nurses on duty who were aware of the intervention, and that may have resulted in assessment bias. Though our study was continued for an hour, in children who benefited from CPAP, this was continued beyond 1 hour, till the clinician decided that CPAP was no longer necessary. Exact data on duration of CPAP was not collected as it was not part of the protocol. We evaluated the respiratory rate only for the first hour; functional outcome such as need for invasive ventilation and duration of hospital stay were not evaluated.

The magnitude of change in the mean RR in our study was much less than that seen in a study from Ghana [4], where children (age 3 months to 5 years) with respiratory distress (including asthma and pneumonia) were treated with CPAP. Among studies that evaluated at the role of CPAP in bronchiolitis, Milesi, et al. [9] found that the use of CPAP decreased inspiratory work in young infants with bronchiolitis. Thia, et al. [10] studied the effect of CPAP on change in partial pressure of $\mathrm{CO}_{2}\left(\mathrm{pCO}_{2}\right)$ in children with bronchiolitis, and observed a significant reduction in $\mathrm{pCO}_{2}$ with use of CPAP when compared to standard care. Machen, et al. [11] also studied the role of CPAP in infants and children with respiratory distress, and documented that CPAP was most beneficial for infants with bronchiolitis. However, a Cochrane review 


\section{What is AlREady KnOWn?}

- CPAP decreases respiratory rate in children with respiratory distress of varied etiology.

\section{What This Study Adds?}

- CPAP is useful in reducing respiratory rate in hospitalized children with bronchiolitis.

suggests that effect of CPAP in children with bronchiolitis is uncertain and larger trials with adequate power are required to further evaluate it [12].

The mechanism by which CPAP works in bronchiolitis is likely to be multifactorial. CPAP works by keeping the airways open, increasing clearance of secretion and improving gas exchange $[13,14]$. Although CPAP has been associated with adverse effects such as nasal mucosal damage, mucosal excoriation, scarring, pressure necrosis, pneumothorax and a decrease in cardiac output [12], none of the patients included in our study had any significant adverse effect.

Our study suggests that CPAP significantly decreases the respiratory rate in patients of acute bronchiolitis in the first hour of treatment. Trials are needed to further investigate if CPAP could be of value in decreasing need of invasive mechanical ventilation and the total duration of hospital stay in children with bronchiolitis.

Contributors: Study was conceived by JP, PB and SNL. The data were collected and analyzed by SNL, JK, PA, KG. The first draft was written by SNL and JP; all the authors approved the final draft.

Funding: None; Competing interest: None stated.

\section{REFERENCES}

1. Ralston SL, Lieberthal AS, Meissner HC, Alverson BK, Baley JE, Gadomski AM, et al. Clinical practice guideline: the diagnosis, management, and prevention of bronchiolitis. Pediatrics. 2014;134:e1474-502.

2. Oymar K, Skjerven HO, Mikalsen IB. Acute bronchiolitis in infants, a review. Scand J Trauma Resusc Emerg Med. 2014;22:3.

3. Beasley JM, Jones SE. Continuous positive airway pressure in bronchiolitis. Br Med $\mathrm{J}$ (Clin Res Ed). 1981;283:1506-8.

4. Wilson PT, Morris MC, Biagas KV, Otupiri E, Moresky
RT. A randomized clinical trial evaluating nasal continuous positive airway pressure for acute respiratory distress in a developing country. J Pediatr. 2013;162: 988-92.

5. Gregory GA, Kitterman JA, Phibbs RH, Tooley WH, Hamilton WK. Treatment of the idiopathic respiratorydistress syndrome with continuous positive airway pressure. N Engl J Med. 1971;284:1333-40.

6. Kaur C, Sema A, Beri RS, Puliyel JM. A simple circuit to deliver bubbling CPAP. Indian Pediatr. 2008;45:312-4.

7. Paediatric Society of New Zealand. 2005. Best Practice Evidenced Based Guidelines. Wheeze and Chest Infection In Infants Under 1 Year. Available from: http://www. paediatrics.org.nz/files/guidelines/Wheezeendorsed. pdf. Accessed February 22, 2017.

8. Verma N, Lodha R, Kabra SK. Recent advances in management of bronchiolitis. Indian Pediatr. 2013;50: 939-49.

9. Milesi C, Matecki S, Jaber S, Mura T, Jacquot A, Pidoux O, et al. $6 \mathrm{cmH} 2 \mathrm{O}$ continuous positive airway pressure versus conventional oxygen therapy in severe viral bronchiolitis: A randomized trial. Pediatr Pulmonol. 2013;48:45-51.

10. Thia LP, McKenzie SA, Blyth TP, Minasian CC, Kozlowska WJ, Carr SB. Randomised controlled trial of nasal continuous positive airways pressure (CPAP) in bronchiolitis. Arch Dis Child. 2008;93:45-7.

11. Machen HE, Mwanza ZV, Brown JK, Kawaza KM, Newberry L, Richards-Kortum RR, et al. Outcomes of patients with respiratory distress treated with bubble CPAP on a pediatric ward in Malawi. J Trop Pediatr. 2015;61:421-7.

12. Jat KR, Mathew JL. Continuous positive airway pressure (CPAP) for acute bronchiolitis in children. Cochrane Database Syst Rev. 2015;1:CD010473.

13. Oymar K, Bardsen K. Continuous positive airway pressure for bronchiolitis in a general paediatric ward; a feasibility study. BMC Pediatr. 2014;14:122.

14. Essouri S, Durand P, Chevret L, Balu L, Devictor D, Fauroux $\mathrm{B}$, et al. Optimal level of nasal continuous positive airway pressure in severe viral bronchiolitis. Intensive Care Med. 2011;37:2002-7. 